\title{
Influence of the Psychophysical Behavior of Pedestrians in the Calibration and Validation of Microsimulation Vissim/Viswalk Models
}

\author{
C. Alcalde, Bachelor of School of Civil Engineering ${ }^{1}$, D. Larrea, Bachelor of School of Civil Engineering ${ }^{1}$, \\ M. Silvera, Master of School of Civil Engineering ${ }^{1}$, and F Campos, Master of School of Civil Engineering ${ }^{1}$ \\ ${ }^{1}$ Universidad Peruana de Ciencias Aplicadas, Perú, u201314698@upc.edu.pe, u201414138@upc.edu.pe, \\ manuel.silvera@upc.edu.pe,pccifcam@upc.edu.pe
}

\begin{abstract}
Pedestrian behavior can be studied force through the social model, which represents the behavior of people through attraction forces that describes the interaction of pedestrians with the environment. These interactions determine the variation of the pedestrian's trajectory, speed, and acceleration. Present investigation seeks to formulate a model of microsimulation software in the Vissim / Viswalk considering pedestrian behavior. The classification of pedestrians' age range is essential for the investigation since it allows to model a behavior closer to reality considering travel speed and trajectory. Results show that tau and social lambda variable force were reduced by $84 \%$ and $15 \%$ respectively. Also, the travel speed of pedestrians concerning the field data without considering the classification exhibited a pedestrian realism of $84 \%$ and $88 \%$ and considering the classification by age range are $97 \%$ the realism. Finally, when considering the psychophysical behavior of pedestrians in the simulation model an increase up to $17 \%$ in the calculation of delay of a road monitoring system is evidenced.
\end{abstract}

Keywords-Pedestrians, social force, microsimulation, Vissim, Viswalk.

\section{INTRODUCTION}

Psychophysics is a discipline of psychology that helps us understand the behavior of individuals under different stimuli. This allows us to study the perceptual movements that made people move and evaluate their behavior taking into account the characteristics of their environment. In recent years, there have been several investigations into the behavior of pedestrians. One reason is the emergence of mathematical models to represent the behavior of pedestrians through the psychophysics. Social force is one such model that was introduced two decades ago by Helbing and Molnár and has since been used in various investigations. The importance of the study of pedestrian simulation models lies in the influence of urban road planning [1]. The case study used is familiar with conflicts that compromise the accessibility and mobility of people and focuses on an intersection that has a composite scenario by a station bus rapid transport (BRT) and informal public transport stops. The latter generated by the lack of infrastructure and adequate signage. This research seeks to analyze how the psychophysical behavior of pedestrians influences the functioning of the road system. As a validation

Digital Object Identifier (DOI):

http://dx.doi.org/10.18687/LACCEI2020.1.1.143

ISBN: 978-958-52071-4-1 ISSN: 2414-6390 tool, this research will develop the microsimulation entire intersection studied using the model of social force in the Vissim/Viswalk software [2]. Calibrated parameters of the model allow representing the actual behavior of pedestrians.

\section{StATE OF THE ART}

The research "Modeling the Variation in the Trajectory of Pedestrians in Crosswalk Overflow Violation China and Countermeasure" behavior of people is evaluated crossing an avenue and conflicts that are generated pedestrians to interact with vehicles are studied. The particularity of this research lies in the variation model pedestrian paths obtained for different scenarios represent overflow the people navigate a crosswalk. The allows to estimate the spatial variations in the paths of people moving out of the zebra type crosswalk [3]. After the analysis concludes that the geometry of the crosswalk, the target type and density of pedestrians are significant factors in the variations of the paths.

The research "In front injured probability assessment pedestrian-vehicle collision counting Uncertainties in pedestrian movement," evaluates a distance model for predicting conflict risks frontal collision between vehicles and pedestrians, it is why it is taken into consideration the probabilistic characteristics mobility [4]. The simulation results show that the probabilistic assessment of the risk of collision between a pedestrian and a vehicle is sensitive to the evasive maneuvers of the driver and provides useful data for optimizing control of conflict prevention. In this paper, we are considering the injuries suffered by pedestrians in the accident. However, authors mention that in a real scenario, you should also consider drivers' injuries in the accidents.

Research on How to Apply Assignment Methods That Were developed for Vehicular Traffic Pedestrian Microsimulations to an allocation method based on traffic time Pedestrians walk is proposed. Authors argue that the choice of route for a pedestrian in a real scenario is based on the shortest walking time and not necessarily the shortest path. To represent this in a micro-iteration were performed by placing various intermediate points along the routes described by pedestrians to calculate the optimal path [5]. In this study, it was demonstrated that a simple allocation method is possible to find a system based on travel time and route choice balance. 


\section{Methodology}

\section{A. Data Collection}

In the city of Lima, Peru, three visits were made to the study area in March on typical days between Tuesday and Thursday, where the system's behavior was analyzed in 8-hour periods. From this study, it was possible to identify that the hour of the greatest presence of pedestrians is from 7 am to $8 \mathrm{am}$.

To represent a pedestrian behavior case study on the microsimulation model was necessary to record a video with the help of a drone. This flew first at a height of 180 meters, intending to find the zones with the most pedestrian density. Here, we're able to identify two areas where most pedestrian activity is presented. These are views are exhibited in Fig. 1.

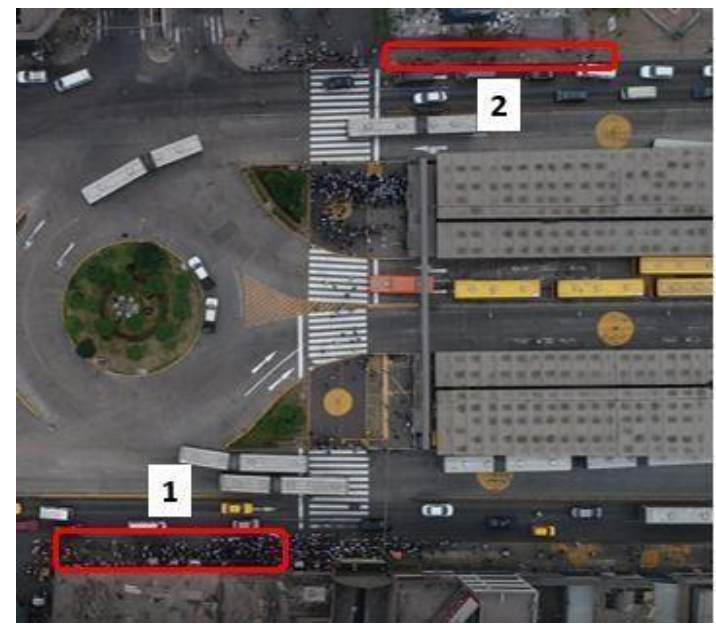

Fig. 1 Above view of the area where the study was conducted.

Through a second drone flight at a height of 40 meters, it can be identified in zone 1 and 2 that pedestrians cannot use the entire width of the sidewalk to move freely. This due to the presence of street vendors who act as obstacles in the path leading to changes and decreased speed of pedestrians. Furthermore, in the 30 meters in length in both areas, the

presence of informal public transport stops is observed. Fig. 2 shows the analysis of the different paths traveled by pedestrians in zone 1. Here you can see the behavior of people, always looking for the easy way, even passing through the middle of the car affecting the efficiency of the system.

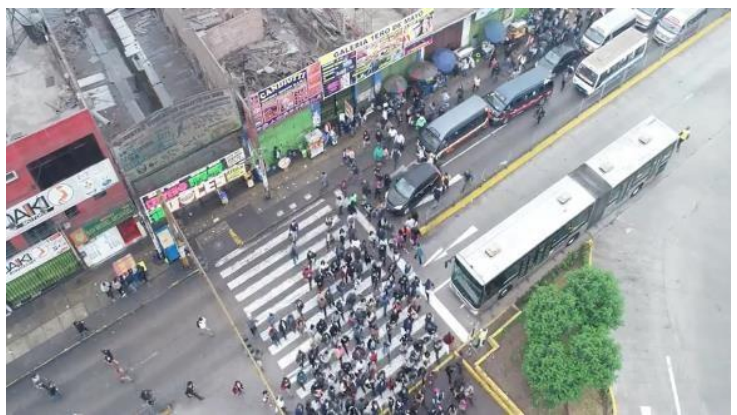

Fig. 2 Above view of the pedestrian's trajectories in zone 1

To ensure that the simulation in the Vissim / Viswalk program can represent behavior that is close to reality, a classification of pedestrians by age range was carried out. In the study area, the age of the pedestrians was determined by viewing the data collection video. With this, the pedestrian capacities were counted taking into account the described trajectory from the informal stops to the crosswalk in both zone 1 and zone 2 .

TABLE I

PEDESTRIAN CAPACITY IN ZONES 1 AND 2

\begin{tabular}{|c|c|c|c|}
\hline $\begin{array}{c}\text { Age range } \\
\text { (years) }\end{array}$ & $\begin{array}{c}\text { Group of } \\
\text { Pedestrians }\end{array}$ & $\begin{array}{c}\text { Zone 1 } \\
\text { (pedestrians / } \\
\text { hour) }\end{array}$ & $\begin{array}{c}\text { Zone 2 } \\
\text { (pedestrians / } \\
\text { hour) }\end{array}$ \\
\hline $0-10$ & I & 0 & 0 \\
\hline 10 to 30 & II & 1096 & 520 \\
\hline $30-50$ & II & 2712 & 1460 \\
\hline $50-$ more & IV & 736 & 196 \\
\hline
\end{tabular}

Table 1 shows that gaging both zone 1 and 2 the range of 0 - 10 and data do not present in the range of 30 - 50 years old is the largest pedestrian concentration. From this information ratio calculated pedestrians in the ranges I, II, III, and IV as displayed in Table 2.

TABLE II

RATIOS FIRST PEDESTRIAN CAPACITY By AGE

\begin{tabular}{|l|c|c|c|c|}
\hline & Range I & Range II & Range III & Range IV \\
\hline Proportion & 0.00 & 0.24 & 0.63 & 0.13 \\
\hline
\end{tabular}

With the classification pedestrian walking speeds are calculated through the measurement of travel times in zones 1 and 2. Table 3 shows some values of speeds obtained for zone 1. The speed of each range was calculated based on 40 samples of pedestrians. 
TABLE III

WALKING SPEEDS IN KM/H IN ZONE 1

\begin{tabular}{|c|c|c|c|}
\hline Range I & Range II & Range II & Range IV \\
\hline- & 8.89 & 5.40 & 5.36 \\
\hline- & 7.05 & 6.04 & 5.10 \\
\hline- & 7.73 & 6.36 & 5.76 \\
\hline- & 7.62 & 5.84 & 5.52 \\
\hline- & 8.31 & 6.21 & 4.88 \\
\hline- & 6.06 & 5.47 & 5.75 \\
\hline- & $7: 45$ & 6.50 & 5.53 \\
\hline
\end{tabular}

With the information of Table 3 accumulated relative frequencies to represent speeds of each group of pedestrians (ranges I, II, III and IV) in the micro-simulation model were calculated. Table 4 shows the calculation of the relative frequencies of the range II accumulated.

TABLE IV

RELATIVE FREQUENCIES ACCUMULATED(H) OF THE RANGE II

\begin{tabular}{|c|c|c|c|c|}
\hline $\begin{array}{c}\text { Intervals } \\
\text { Range II }\end{array}$ & $\mathrm{F}$ & $\mathrm{F}$ & $\mathrm{h}$ & $\mathrm{H}$ \\
\hline 6.5 & 1.0 & 1.0 & 0.04 & 0.04 \\
\hline 7.0 & 2.0 & 3.0 & 0.08 & 0.12 \\
\hline 7.5 & 7.0 & 10.0 & 0.28 & 0.40 \\
\hline 8.0 & 9.0 & 19.0 & 0.36 & 0.76 \\
\hline 8.5 & 5.0 & 24.0 & 0.20 & 0.96 \\
\hline 9.0 & 1.0 & 25.0 & 0.04 & 1.0 \\
\hline
\end{tabular}

As additional information for developing the model, Vissim / Viswalk generated conflict points identified by the rise and fall of passengers in informal stops as shown in Fig. 3. These points were recognized from field observations.

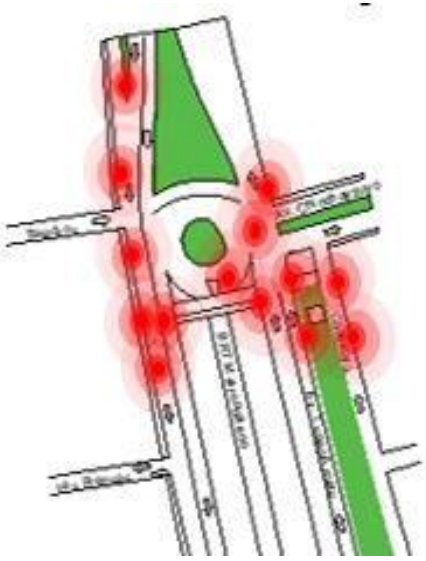

Fig. 3 Identification of conflict spots in informal whereabouts

\section{B. Model generation in Vissim/Viswalk}

The Vissim / Viswalk software was used to generate the microsimulation model of the $6800 \mathrm{~m}^{2}$ of the study area. Within the model, the different trajectories of the people as visualized in the aerial shot of the drone were represented. This allowed generating the movement of pedestrians along the sidewalk and in the middle of the main avenue as shown in Fig. 4.

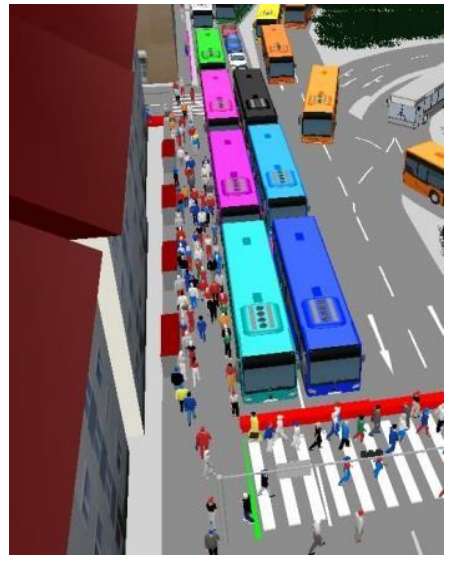

Fig. 4 Pedestrians displacement in Zone 1

Relative frequencies accumulated in Table 4 helped to build graphs depicting speeds in zone 1 and 2 within the Vissim / Viswalk software. Significantly speed distributions were developed for ranges II, III and IV as for the range I no data recorded. Fig. 5 shows the distribution of speed range of group II representing the highest walking speed.

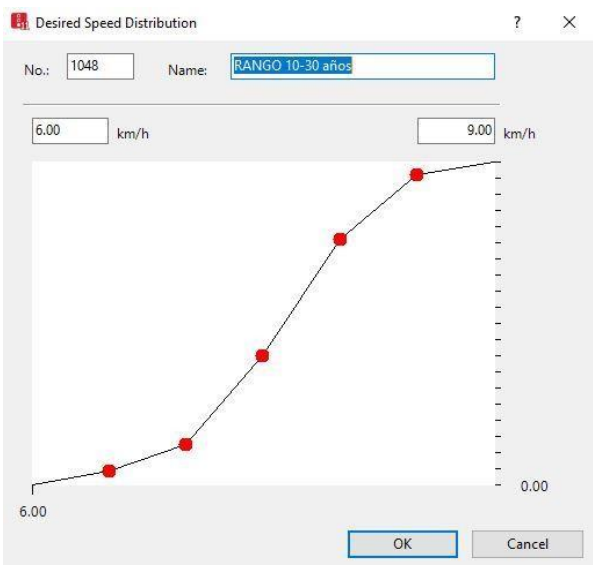

Fig. 5 Distribution Speed Range 10 to 30 years

Also, a graph of speed distribution was carried out without considering the age ranges within a second microsimulation model to be able to perform a comparative analysis as shown in Fig. 6.

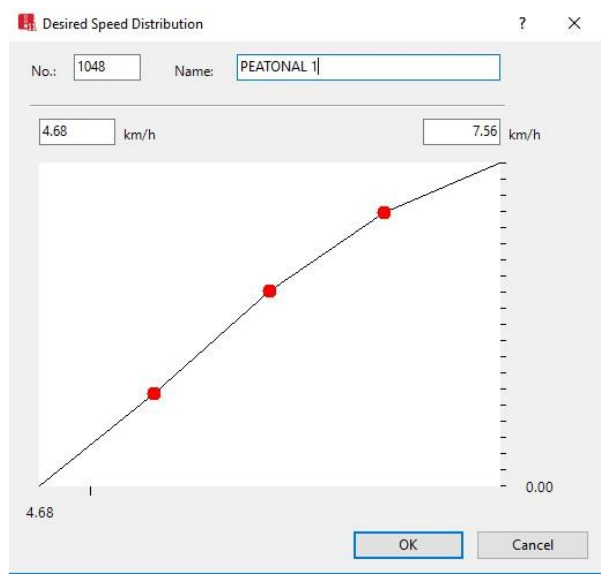

Fig. 6 Distribution Speed Without considering Age as a Factor 


\section{Social Force Model}

The Vissim / Viswalk software includes three variables of the model of social force that complement the analysis of the behavior of pedestrians with their environment. Tau variable $(\tau)$ associated with acceleration or pedestrians' reaction and lambda $(\lambda)$ representing the influence of the pedestrians on a person walking and noise associated with the randomness of the results.

Given the influence of each variable on the behavior of pedestrians within the microsimulation model, it is intended that values allow making a similar representation to reality [6].

\section{RESULTS}

In this part of the article presents the main results of the research are presented.

Table 5 shows the final values of the parameters of social force tau, lambda, and noise obtained after 12 iterations.

TABLE V

PARAMETER VALUES OF SOCIAL ForCE

\begin{tabular}{|c|c|c|c|}
\hline & Tau & lambda & Noise \\
\hline Values & 0.065 & 0.2 & 1.2 \\
\hline
\end{tabular}

These values will be used in the micro-simulation model to represent the actual behavior of pedestrians in this intersection for the case study.

Then, 15 runs were carried out in the Vissim/Viswalk software, obtaining reports speed in zones 1 and 2. In Table 6, an example of the first seven pedestrian's speed route were obtained from the reports of the software considering age rating of pedestrians.

TABLE VI

FIRST I TRAVEL SPEED REPORT VISSIM/VISWALK CONSIDERING AGE RANGE

\begin{tabular}{|c|c|c|}
\hline Counting & Zone 1 & Zone 2 \\
\hline 1 & 6,038 & 5,964 \\
\hline 2 & 6,056 & 5,936 \\
\hline 3 & 6,053 & 5,970 \\
\hline 4 & 6,027 & 5,953 \\
\hline 5 & 6,021 & 6,015 \\
\hline 6 & 6,029 & 5,961 \\
\hline 7 & 6,052 & 5,983 \\
\hline
\end{tabular}

For comparative analysis speed reports were also generated in Vissim/Viswalk software without taking into consideration the classification of pedestrians age described in Table 1. This analysis seeks to identify what is the influence of the psychophysical behavior of people within microsimulation and the road system. Table 7 exhibit the results obtained.
TABLE VII

VISSIM TRAVEL SPEED WiTHOUT CONSIDERING AGE RANGE

\begin{tabular}{|c|c|c|}
\hline Counting & Zone $1(\mathrm{~m} / \mathrm{s})$ & Zone $2(\mathrm{~m} / \mathrm{s})$ \\
\hline 1 & 5,373 & 5,163 \\
\hline 2 & 5,454 & 5,212 \\
\hline 3 & 5,490 & 5,153 \\
\hline 4 & 5,460 & 5,185 \\
\hline 5 & 5,428 & 5,145 \\
\hline 6 & 5,459 & 5,183 \\
\hline 7 & 5,499 & 5,203 \\
\hline
\end{tabular}

Then the average speed from Table 6 and 7 was calculated. Values are compared with the rates obtained in the field to evaluate the margin of error as shown in Table 8.

TABLE VIII

RESUlTS COMPARISON OF PEDESTRIAN'S AVERAGE SPEED

\begin{tabular}{|c|c|c|c|}
\hline Zone & Field & Age-rated $(\mathrm{m} / \mathrm{s})$ & No age rating $(\mathrm{m} / \mathrm{s})$ \\
\hline 1 & 6.21 & 6.04 & 5,452 \\
\hline 2 & 6.14 & 5,967 & 5,178 \\
\hline
\end{tabular}

Analyzing the results of Table 9 using the pedestrian classification by age range microsimulation model exhibits a similarity of $97 \%$ compared with the field data. Instead, the second model that does not consider the classification of pedestrians resembles field data by $84 \%$ in zone 1 and $88 \%$ in zone 2 .

The efficiency of the intersection can be measured through the delay control. The Vissim software using the node command lets you calculate different variables that define the efficiency of the system. Among these, we can mention the average delay, the maximum delay, average, and maximum vehicle queues. For our research node, such command was used as shown in Fig. 7.

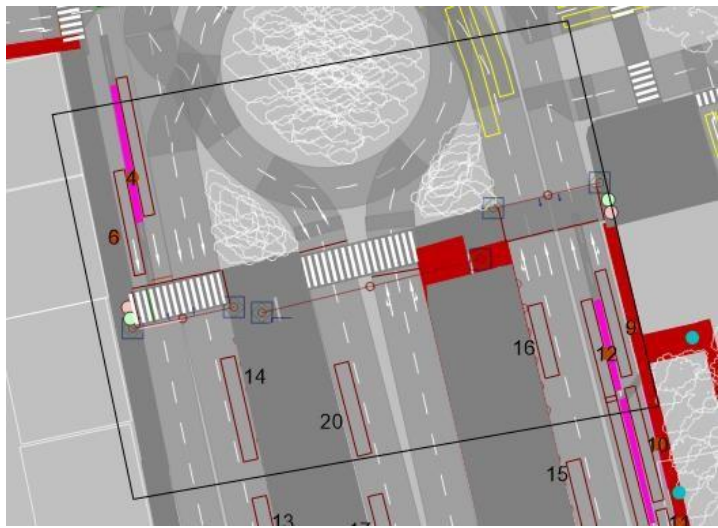

Fig. 7 Knot at the Intersection

With this command, we obtained the reports of 15 intersections control delays that served to calculate the average delays in seconds/pedestrian for each zone, as detailed in Table 9 . 
TABLE IX

AVERAGE DELAY OF THE INTERSECTION

\begin{tabular}{|l|c|c|}
\hline & $\begin{array}{c}\text { With pedestrian } \\
\text { classification (s) }\end{array}$ & $\begin{array}{c}\text { Regardless } \\
\text { classification (s) }\end{array}$ \\
\hline Zone 1 & 227.94 & 207.10 \\
\hline Zone 2 & 166.13 & 142.05 \\
\hline
\end{tabular}

The results in Table 9 show psychophysical behavior as represented by the simulation of the paths of pedestrians and classifying people by age range, directly influence the efficiency and operation of the road system.

\section{VALIDATION}

On the recommendation of the Federal Highway Administration (FHWA), the results of microsimulation models must be calibrated and validated to ensure that the output represent reality [7].

In this research the calibration of the model was performed by comparing the data recorded from field and the Vissim's speeds, considering the classification by age range and pedestrian traffic counts. The calibration tool as a statistical difference test was conducted in the Statkey program [8].

In zone 1 the mean difference in speed is 0.17 . Fig. 8 shows normal distribution generated in Statkey program.

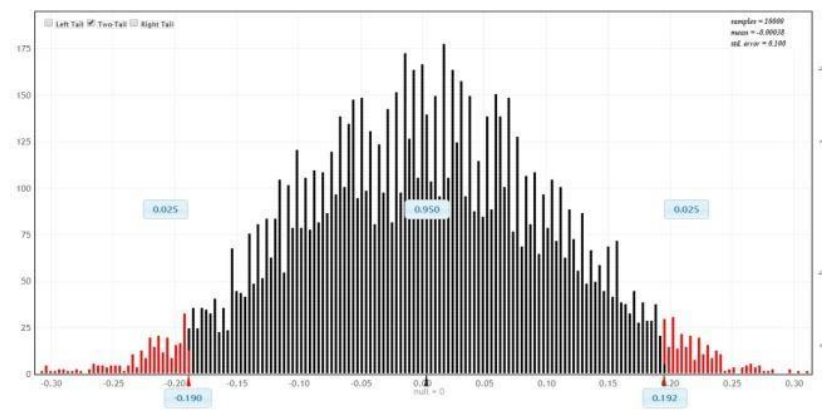

Fig. 8 Knot at the Intersection

This figure shows that the acceptance limits are between 0190 and 0192 . With these values, we can say that the mean difference is in the distribution and thus the null hypothesis is accepted. The same process was performed in zone 2 and the results were also accepted. With this statistical test shows that the microsimulation model is calibrated.

For the validation process of the model, a second data collection was conducted a week after the first aerial footage of the drone, respecting the time where the maximum volume of pedestrians is presented. Here the field data collection was performed again to calculate new proportions to be considered in classifying pedestrians. Table 10 shows the new data in the proportion of pedestrian zones 1 and 2 .

TABLE X

AVERAGE DELAY OF THE INTERSECTION

\begin{tabular}{|c|c|c|c|c|}
\hline & Rank 1 & Rank 2 & Rank 3 & Rank 4 \\
\hline Proportion & 0.00 & 0.25 & 0.63 & 0.12 \\
\hline
\end{tabular}

For the validation process, the same considerations described the number of pedestrian samples and calculation speeds field to zones 1 and 2. Within the micro-simulation model was taken of the methodology with respect built again graphics velocity distribution. Fig. 9 exhibits the new velocity distribution calculated for range III and the second field data collection.

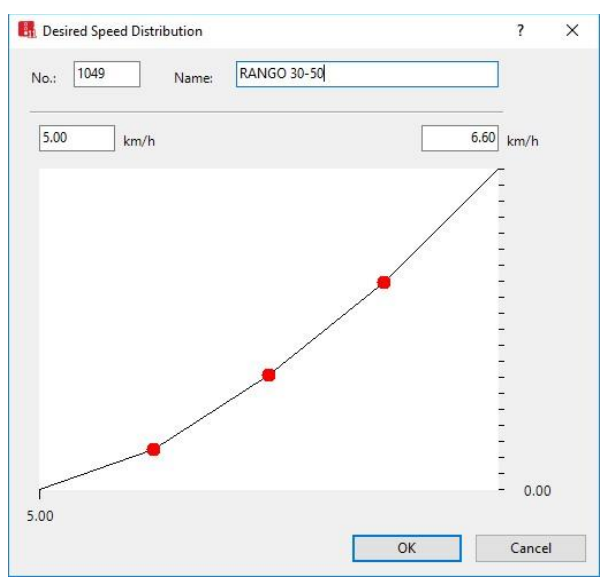

Fig. 9 Speed distribution for age range III

Within the micro-simulation model, the same values of variables social strength are shown in Table 6 . The following were considered, 15 runs the model that allowed obtaining reports speed for zones 1 and 2. The table was performed 12 shows as an example the first 7 results of the reports Vissim/Viswalk program.

TABLE XI

SECOND SPEED REPORT FROM VISSIM/VISWALK RECITAL AGE RANGE FOR THE VALIDATION MODEL

\begin{tabular}{|c|c|c|}
\hline Counting & Zone 1 $(\mathrm{m} / \mathrm{s})$ & Zone 2 $(\mathrm{m} / \mathrm{s})$ \\
\hline 1 & 6,038 & 5,964 \\
\hline 2 & 6,056 & 5,936 \\
\hline 3 & 6,053 & 5,970 \\
\hline 4 & 6,027 & 5,953 \\
\hline 5 & 6,021 & 6,015 \\
\hline 6 & 6,029 & 5,961 \\
\hline 7 & 6,052 & 5,983 \\
\hline
\end{tabular}

For the calibration process the reports generated by the software were analyzed, resulting in a statistical mean difference. In zone 1 the mean difference velocity is 0.12 . In Fig. 10 normal distribution graph generated in the Starkey program is shown. 


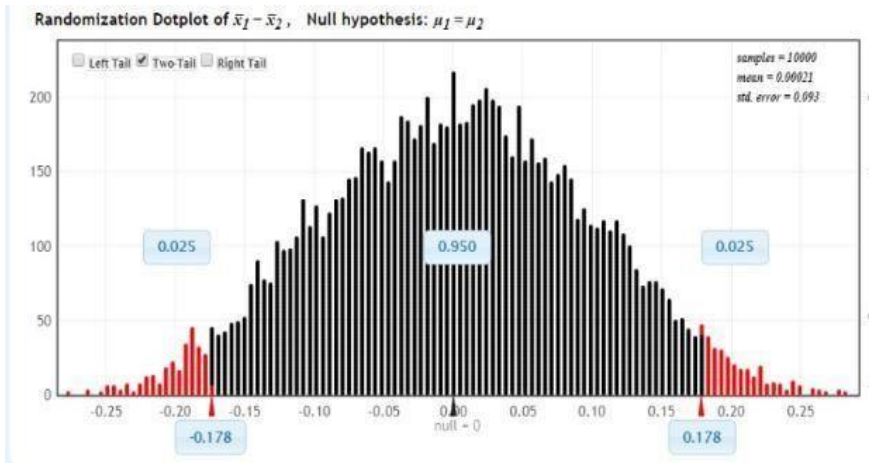

Fig. 10 Distribution of null hypothesis in zone 1

Fig. 10 shows that the acceptance limits are between 0178 and 0178 . With these values, we can say that the mean difference is in the distribution and thus the null hypothesis is accepted. The same process was performed in zone 2 and the results were also accepted. With this statistical test shows that the microsimulation model is validated.

\section{CONCLUSIONS}

The parameters of social strength were obtained: tau $=$ 0.065 , lambda $=0.15$ and noise $=1.20$. These values allowed calibrate and validate the model on the recommendation of the FHWA, simulating the actual behavior of zones 1 and 2 .

Results were compared with two simulation models, one considering the psychophysical analysis of pedestrians and the other without this behavior. With these results, it is seen that the simulation of the paths of pedestrians and classifying people by age range generates a similarity of $97 \%$ with field data of zones 1 and 2. However, in the second model where no psychophysical behavior it considered the results show a similarity of $84 \%$ in zone 1 and $88 \%$ in zone 2 .

A psychophysical considering the behavior of pedestrians in the simulation model, an increase of up to $17 \%$ is shown by the calculation delays of the road system. This result was compared with the second simulated model, which does not contemplate walking path analysis and classification by age range of people.

With these results the importance of considering the psychophysical analysis of pedestrians in urban planning of a city is evident. Because this behavior influences the efficiency of the road system and infrastructure design consideration pedestrian accessibility.

\section{REFERENCES}

[1] F. Johanssona, A. Peterson y A. Tapani, "Waiting pedestrians in the social force model", Physica A, vol. 419 (2015), pp 95-107.

[2] Planung Transport Verkehr (2019). Vissim (Versión 9) [Software]. Available: https://www.ptvgroup.com/es/soluciones/productos/ptv-vissimnuevo/

[3] C. Ning-Bo, Q. Zhao-Wei., S. Xian-Min, Z. Li-Ying, B. Qiao-Wen, y L. Rui-Qi, "Modeling the Variation in the Trajectory of Crosswalk Overflow Violation Pedestrians in China and Countermeasure. Mathematical Problems in Engineering", Hindawi, vol. 2017, pp. 1-14. Agosto 2017.

[4] Z. Huang, Y. He, Y. Wen y X. Song, "Injured probability assessment in frontal pedestrian-vehicle collision counting uncertainties in pedestrian movement", Safety Science, vol. 106, pp. 162-169. Julio 2018.
[5] V. Roca, V. Torres, T. Kretz, K. Lehmann y I. Hofsab, "How to Apply Assignment Methods that were Developed for Vehicular Traffic to Pedestrian Microsimulations", Procedia - Social and Behavioral Sciences, vol. 162, pp. 15-23. Diciembre 2014

[6] L. Ma, B. Chen, X. Wang, Z. Zhu, R. Wang X. Qiu. "The analysis on the desired speed in social force model using a data driven approach", Physica A, vol. 525, pp. 894-911. Julio 2019.

[7] Federal Highway Administration (2019, Junio 20). Calibration of Microsimulation Models [Online]. Available: https://ops.fhwa.dot.gov/trafficanalysi stools/tat_vol3/sect5.htm

[8] Statkey (2019, 25 de junio). Test for difference in means. Available: http://www.lock5stat.com/StatKey

[9] Planung Transport Verkehr (2019, 10 de abril). Manual de Vissim 9.Available: https://www.scribd.com/document/353480439/Vissim-9-Manual

[10] Anvari, M.Bell, A. Sivakumar y W. Ochieng. "Modelling shared space users via rule-based social force model", Transportation Research Part C, vol. 51, pp. 83-103. Octubre 2014

[11] A. Shalom H., V. Gitelman, E. Ben-Shabat. "An evaluation of crosswalk warning systems: effects on pedestrian and vehicle behaviour", Transportation Research Part F, vol. 5, pp. 275-292. Junio 2002.

[12] I. Kaparias, M. Bell, J. Greensted, S. Cheng, A. Miri, C. Taylor y Bill Mount. "Development and Implementation of a VehiclePedestrian Conflict Analysis Method", Transportation Research Board, vol. 2196, pp. 75-82. 